\title{
Isolation and characterization of floral transcripts from mangosteen (Garcinia mangostana L.).
}

\begin{abstract}
The understanding of flower initiation, development, and maturation in mangosteen is of paramount importance to shorten its long juvenile phase and to synchronize its flowering or fruiting time. In this study, we have identified 97 tentative unique genes with higher expression levels in young flower buds compared to young shoots by using suppressive subtraction hybridization and reverse northern analysis. Sequence analysis showed that $63.9 \%$ of these transcripts had non-significant matches to sequences in the non-redundant protein database in Gen- Bank, $19.6 \%$ had significant matches to unknown proteins while the remaining $16.5 \%$ had putative functions in transcription, stress, signal transduction, cell wall biogen-esis, photosynthesis and miscellaneous. The full-length cDNA of GmAGMBP encoding AG-motif binding protein(a zinc finger transcriptional factor), and 30 termini cDNA sequences of GmHSA32 and GmBZIP, encoding heat-stress-associated 32 (HSA32) and bZIP transcription factor,respectively; were cloned and further analysed. Real-time PCR analysis revealed that these three genes have different transcript profiles in flowers of different developmental stages and young shoots. The highest abundance of transcripts was achieved in flowers with diameters ranging from 0.5 to $0.9 \mathrm{~cm}$ for GmAGMBP and GmBZIP and in flowers with diameters less than $0.5 \mathrm{~cm}$ for GmHSA32. Southern analysis suggested that GmAGMBP might be single copy gene while GmHSA3A could possibly belong to a small gene family in the mangosteen genome.
\end{abstract}

Keyword: Flower; Mangosteen; Real-time PC; Suppressive subtraction hybridization. 